\title{
Sarcoglycanopathy - A Rare Case Report and Literature Review
}

\author{
EKRAMUL MUSTAFA, ${ }^{1}$ MD. AZIZUL HASAN KHANDAKER,${ }^{2}$ MD. MAMUNUR RASHID, ${ }^{3}$ SWAPON KUMAR GHOSE, ${ }^{4}$ \\ MOSTOFA KAMAL CHOWDHURY 5
}

\begin{abstract}
:
Sarcoglycanopathies are relatively rare progressive muscular dystrophies with autosomal recessive inheritance designated as $\alpha, \beta$, $\gamma$, or $\delta$ sarcoglycanopath.; which belong to the group of limb girdle muscular dystrophies (LGMD) and are caused by mutations in any of the four sarcoglycan genes: alpha (LGMD 2D), beta (LGMD 2E), gamma (LGMD 2C) and delta (LGMD 2F). The phenotype resembles dystrophinopathies due to proximal muscle weakness and calf hypertrophy. Reports from Bangladesh are scarce. We report a rare case of primary sarcoglycanopathy (SGP) which emphasizes the evolving concept of "dystrophinopathy to sarco-glycanopathy" and describe literature pertaining to this rare entity.
\end{abstract}

Key words: Sarcoglycanopathy; Dystrophinopathy; Limb girdle muscular dystrophies.

\section{Introduction:}

Limb girdle muscular dystrophy (LGMD) has long been a controversial entity. ${ }^{1,2}$ Limb girdle muscular dystrophies (LGMD) are genetically heterogeneous group of disorders, characterized clinically by predominant proximal muscle weakness of variable severity, elevated creatinine kinase levels and dystrophic changes on muscle biopsy ${ }^{3}$ The dominant forms are generally milder and relatively rare, representing less than $10 \%$ of all LGMD. The autosomal recessive forms are more common, having a cumulative prevalence of $1: 15,000 .{ }^{4}$ The sarcoglycans are a group of 4 distinct integral membrane proteins, each from a distinct gene, that associate in a tetrameric complex, known as sarcoglycan complex. The complex is directly associated with dystroglycan and indirectly with dystrophin. ${ }^{5}$ There are four sarcoglycan genes and their corresponding proteins, namely $\mathrm{a}, \mathrm{b}, \mathrm{g}$, and $\mathrm{d}$. These are transmembrane glycoproteins and range in size from 35 to $50 \mathrm{KD}$. The a,b,c,d, saroglycan complex is muscle-specific, however, a fifth protein e-sarcoglycan replaces a-sarcoglycan in some non-striated muscle tissues. Mutations in any one of the genes except epsilon result in autosomal recessive limb girdle muscular dystrophy (LGMD $2 \mathrm{C}-2 \mathrm{~F}$ ) which is clinically and pathologically indistinguishable from primary dystrophinopathies. ${ }^{6}$ The function of the sarcoglycan complex is not exactly known. However, they

1. Lecturer, Department of Physiology, Dhaka Medical College

2. Consultant (Medicine), Upazilla Health Complex, Adamdighi, Bogra.

3. MD student, Neurology, Dhaka Medical College \& Hospital

4. MD ( Neurology), Dhaka Medical College \& Hospital

5. Indoor Medical Officer, Department of Medicine, Dhaka Medical College \& Hospital

Correspondence: Dr. Ekramul Mustafa, Lecturer, Department of Physiology, Dhaka Medical College. Mob: 01712005996, E-mail:drekram55@gmail.com. are thought to be involved in the stabilization of the membrane and signal transduction.

Individually, these subtypes are difficult to distinguish and require immunocytochemistry, immunoblotting or molecular methods. Reports of these have appeared from European countries, Japan, United States and North America. Reports from the Indian subcontinent are scarce and recent. ${ }^{7,8}$ Here, we report and discuss the presentation of a case of primary beta-sarcoglycanopathy which is extremely rare.

\section{Case study:}

This six and half years old female child, normotensive, nondiabetic, born of non-consanguineous marriage, full term normal delivery, without perinatal hypoxia with normal developmental milestones presented with weakness and wasting of both Lower Limbs. This was insidious in onset and gradually progressive. Predominant involvement was proximal than distal muscle group. She started having difficulty in walking first, then complained of frequent tripping on uneven surfaces and difficulty to get up from supine position. No history of slippage of sandals or foot drop. Patient is ambulatory without support. She also developed calf hypertrophy. No history of weakness in upper limbs, sphincteric involvement, sensory involvement, back or neck pain. Cranial nerves were normal. Prior fever, trauma, headache, loss of consciousness and seizures were absent. No significant family history. General examination revealed patient was ill looking, oriented, afebrile, pulse- 86 beats/ min, regular, all peripheral pulses present, Blood Pressure110/70 mm of Hg. No clubbing, cyanosis, jaundice, edema, pallor or lymphadenopathy.

Neurological examination revealed conscious, oriented and with normal speech, cranial nerves were intact including both fundi. Atrophy was present in quadriceps bilaterally. Bulk 
was also decreased in proximal lower limbs, calf, infraspinatus, however, extensor digitorum brevis were hypertrophied., Tone was decreased in lower limbs. Power was $4 / 5$ in lower limbs proximally and 5/5 distally, trunkal weakness was also present. Power of upper limbs was normal in proximally and distally. Deep tendon reflexes were normal, abdominal reflex present, Plantar reflex bilaterally flexor, all modalities of sensory functions were normal, cerebellar signs were normal, gait revealed toe walking present with hyperextension at knee and Gowers sign, Valley sign-positive . No signs of meningeal irritation. Cranium and spine were normal. Other systemic examination revealed no abnormality.

The anatomical localization was either anterior horn cell (unlikely as the weakness and wasting was symmetrical and progressive without fasciculations), radicals (unlikely as there is no radicular pain), nerves (may be present-flaccid quadriparesis with diminished jerks but then there is global weakness also involving distal extremity, normal NCV is also against it), or neuromuscular junction (unlikely with no diurnal variation), or myopathy (likely with insidious progression and symmetrical proximal involvement). The etiological possibilities that were kept Duchenne muscular dystrophy and LGMD based on the clinical pattern and raised CPK level and EMG findings.

Investigations revealed $\mathrm{Hb}-12.1 \mathrm{gm} / \mathrm{dl}$, total count of $\mathrm{WBC}$ $8000 / \mathrm{mm}^{3}$, Neutrophil 70\%, Lymphocyte 26\%, Monocyte, Eosinophil \& Basophil 3\%) Platelets-normal, ESR-22 mm in the first hour. FBS-94.8 mg\% Blood Urea/Serum Creatinine18.5/0.69 mg\%, serum Na/K-139.4/4.57 mEq/L, Bilirubin$0.44 \mathrm{mg} \%$, Total Protein-6.61 gm $\%$, Albumin-4.11gm $\%$, ALT/ AST-120.07/42.2 U/L. HIV 1 \& 2-non reactive. RA/ANA testNegative, ECG-Normal, MRI of cervical spine \& CT headNormal, CPK-5934 IU, EMG/NCV-suggestive of myopathic pattern with normal NCS. Muscle biopsy revealed Paraffin and cryosections from skeletal muscle show partly effaced architecture. There is moderate degree of endo and perimysial fibrosis and adipose tissue infiltration. Myofibrils are round, vary markedly in diameter with a few angulated atrophic fibers. Central nuclei, necrosis, amyophagocytosis and clusters of regenerating fibres are evident. One focus of perimysial perivascular inflammation is seen. Moth eaten/ lobulated fibers are not observed. ATPase reveals type I and II atrophy. Focal type-II fiber grouping, while mosaic pattern is maintained in rest of the fascicle. Muscle biopsy specimen for further molecular studies were sent and Immunostaining to sarcoglycans $(\alpha, \beta, \gamma$, or $\delta$ ) shows total absence in all fibers. Dysferlin is preserved (Fig-1). Muscle biopsy specimen were sent for genetic analysis but report was not collected for financial constrain. DMD gene deletion studies using multiplex PCR were not contributory.
We concluded that this was a case of sarcoglycanopathy. Physiotherapy was started, calcium, vitamin-D and antioxidant supplement were given. She is now on follow up.

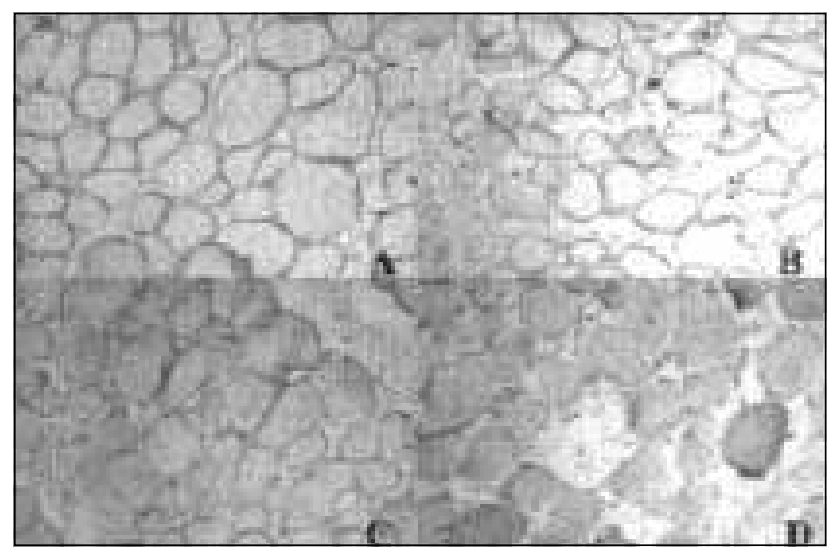

Fig.-1. Photograph showing positivity for dystrophin $(A, \times$ 100), alpha-sarcoglycan $(B, \times 100)$, beta-sarcoglycan $(C, \times 100)$ and negative staining for gamma-sarcoglycan $(D, \times 100)$.

\section{Discussion}

The prevalence of the autosomal recessive LGMD is estimated to be about I in $15,000 .{ }^{4}$ Sarcoglycanopathies (SG) form an important subgroup which are distinct from dystrophinopathies (DP). The common feature of SG is preferential and early involvement of the lower girdle muscles, subsequent involvement of shoulder girdle with scapular winging. The distal group of muscles is either not affected or affected less (typically). There is usually no involvement of facial and ocular muscles. An early onset dystrophy or late onset LGMD should alert the clinician to induce appropriate studies of protein and DNA studies to diagnose a SG. The clinical spectrum can be heterogeneous with some cases having minimal muscle involvement revealed by increased CPK levels to some having a severe phenotype and being wheelchair bound by the second decade.

In contrast to DP, sarcoglycanopathies are characterized by the absence of mental retardation and overt cardiac involvement. The authors are unable to explain the speech delay in the present case. Since sarcoglycan is ubiquitous in its presence, being present in cardiac muscle as well, reports of cardiomyopathy and recurrent hemoglobinuria have appeared in literature. ${ }^{9,10}$ The first reported case of SG was a 3-year-old girl described by Bonnemann et al ${ }^{11}$

Diagnosis is usually made by muscle biopsy with specific immunohistochemical staining with monoclonal antibodies to specific proteins. Testing for dystrophin gene or protein 
has become a part of the routine diagnostic evaluation of patients who present with proximal muscle weakness. In addition to dystrophin, dystrophin associated proteins like dystroglycan, sarcoglycan and synaptaphorin contribute to the membrane exoskeleton. Each of the sarcoglycan is a small transmembrane protein and can be identified on staining with antibodies directed against á,â,ä and ã sarcoglycan. Mutations in a single sarcoglycan gene lead to destabilization of the entire sarcoglycan complex and secondary deficiency of other sarcoglycan protein. Since biopsy specimens from patients with dystrophinopathy reveal secondary deficiency of sarcoglycan proteins, testing for these sarcoglycan proteins is informative only if dystrophin is normal. Immunocytochemistry helps in delineating the type of SG. ${ }^{12}$

Molecular studies, wherever available, help in confirming the diagnosis. For genetic study sample has been send but report is not collected for financial constrain. The initial mutation identified was a truncating mutation in the $43 \mathrm{kDa}$ fraction in a sporadic case of LGMD2E. ${ }^{13}$ Later missense mutations were identified in Indiana Amish pedigrees. ${ }^{14,15}$ Most of the other mutations identified are missense, some are frame shift. Those with missense mutations present with a mild phenotype and those with frame shift with a severe phenotype. This suggests that the part of the gene in which the mutations are found (immediately behind the transmembrane domain) may be particularly sensitive to the effects of mutations which might affect the secondary structure of protein. ${ }^{11}$

\section{Conclusion}

Though sarcoglycanopathy is rare, it also should be suspected in a child with early childhood onset, proximal muscle weakness and calf hypertrophy, raised CPK and a myopathic pattern on the EMG. Haplotype analysis at autosomal recessive LGMD loci may be informative but immunocytochemistry using dystrophin and sarcoglycan antibodies usually establishes the diagnosis in the absence of molecular testing. Genetic counseling is important due to its amenability to prenatal diagnosis even though here positive family history was absent.

\section{Conflict of Interest : None}

\section{References}

1. Bradley WG. The limb girdle syndromes. In: Vinken PJ, Bruyn GW, editors. Handbook of Clinical Neurology, Vol 40: Amsterdam: Elsevier; 1979; 433-69.

2. Shields RW Jr. Limb girdle syndromes. In: Engel AG, Franzini AC, editors, Myology. 2nd Ed. New York: McGraw Hill; 1994;1258-74.
3. Cagliani R, Comi GP, Tancredi L, Sironi M, Fortunato F, Giorda R et al. Primary betasarcoglycanopathy manifesting as recurrent exercise induced myoglobinuria. Neuromuscul Disord 2001; 11(4): 389-394.

4. Nigro V. Molecular bases of autosomal recessive limb-girdle muscular dystrophies. Acta Myo12003; 22(2): 35-42

5. Hoffman EP. Sarcoglycanopathies. In: George Karpati, editors, Pathology and Genetics Structural and Molecular Basis of Skeletal Muscle Diseases. Basel: ISN Neuropath Press; 2002;24-7.

6. Duggan DJ, Gorospe JR, Fanin M, Hoffman EP, Angellini C. Mutations in the sarcoglycan genes in patients with myopathy. N Engl J Med 1997; 336:618-24.

7. Khadilkar SV, Singh RK, Katrak SM. Sarcoglycanopathies : a report of 25 cases. Neurol India 2002; 50(1): 27-32.

8. Gulati S, Leekha S, Sharma MC, Kalra V. Gammasarcoglycanopathy. Indian Pediatr 2003; 40(11): 1077- 1081.

9. Fanin M, Melacini P, Boito C, Pegoraro E, Angelini C. LGMD2E patients risk developing cardiomyopathy. Neuromuscul Disord 2003; 13(4) : 303-309

10. Sylvius N, Duboscq-Bidot L, Bouchier C, Charron P, Benaiche A, Sebillon $\mathrm{P}$ et al. Mutational analysis of the beta- and deltasarcoglycan genes in a large number of patients with familial and sporadic dilated cardiomyopathy. Am J Med Genet 2003; 120 (1) : 8-12.

11. Bonnemann CG, Modi R, Noguchi S, Mizuno Y, Yoshida M, Gussoni E et al. Betasarcoglycan (A3b) mutations cause autosomal recessive muscular dystrophy with loss of sarcoglycan complex. Indian Journal of Pediatrics, Volume 72 January, 200573 Seema Kapoor et al. Nat Genet 1995; 11 (3): 266-273.

12. Ogawa E, Yoshida M, Suzuki A, Mizuno Y, Hagiwara Y, Noguchi S. Dystrophin associated proteins in muscular dystrophy. Ttuman Mol Genet 1995; 4: 1711-1716.

13. Lim LE, Duclos F, Broux O, Bourg N, Sunada Y, Allamand Vet al. Betasarcoglycan: characterization and role in limb girdle muscular dystrophy linked to 4q12. Nat Genet 1995; 11(3): 257- 265.

14. Bonnemann CG, Wong J, Ben Hamida C, Hamida MB, Hentati F, Kunkel LM. LGMD2E in Tunisia is caused by homozygous missense mutations in betasarcoglycan exon 3 . Neuromuscul Disord 1998; 8(4) : 193-197.

15. Duggan DJ, Gorospe JR, Fanin M, Hoffman EP, Angelini C. Mutations in sarcoglycan genes in patients with myopathy. N Engl J Med 1997; 336(9): 618-624. 